\title{
Les murs comme support du politique : la brigade Chacón au Chili (1989-1997)
}

\author{
Antonia GARCIA CASTRO
}

\section{OpenEdition}

1 Journals

\section{Édition électronique}

URL : http://journals.openedition.org/conflits/1848

DOI : $10.4000 /$ conflits.1848

ISSN : $1777-5345$

Éditeur :

CCLS - Centre d'études sur les conflits lilberté et sécurité, L'Harmattan

\section{Édition imprimée}

Date de publication : 1 mars 2005

Pagination : 259-275

ISBN : 2-7475-8103-9

ISSN : 1157-996X

\section{Référence électronique}

Antonia GARCIA CASTRO, « Les murs comme support du politique : la brigade Chacón au Chili (1989-1997) », Cultures \& Conflits [En ligne], 57 | printemps 2005, mis en ligne le 10 janvier 2006, consulté le 30 mars 2021. URL : http://journals.openedition.org/conflits/1848 ; DOI : https://doi.org/ $10.4000 /$ conflits. 1848

Ce document a été généré automatiquement le 30 mars 2021.

Creative Commons License 


\title{
Les murs comme support du politique : la brigade Chacón au Chili (1989-1997)
}

\author{
Antonia GARCIA CASTRO
}

« La gloire de ce peintre est invulnérable, car (...) des tableaux qu'il a exécutés, il n'en reste plus aucun ». Jorge Luis Borges ${ }^{1}$

Le présent dossier porte son attention sur une modalité paradoxale de la prise de parole : parole muette, peinte sur un mur, que donne à voir la brigade Chacón au Chili. Cette expérience a des précédents que l'on examine avant d'en venir aux caractéristiques spécifiques de la brigade choisie. Le témoignage d'une brigadiste - et, en annexe, quelques propos du peintre Roberto Matta - complètent ce dossier dans sa version papier ${ }^{2}$. Cette expérience nous renvoie certes à une histoire spécifique. En l'occurrence à une histoire chilienne. Elle est aussi symptomatique d'un phénomène plus large: les nombreux liens de l'image et du politique; et, dans le cas précis, ceux de la peinture et du politique ${ }^{3}$. Que la parole soit censurée, ou qu'elle s'avère impropre, le tracé participe parfois de la contestation. Parole muette donc, non dépourvue de sens.

Emergence des brigades murales au Chili ${ }^{4}$

2 Surgies à la fin des années 1960, les brigades murales acquièrent une visibilité à l'occasion de la campagne présidentielle de 1970. Les brigades sont alors affiliées à des partis politiques, elles sont constituées de militants - jeunes pour la plupart; et si l'ensemble du spectre politique chilien se dote à un moment donné de sa brigade, l'émergence du travail muraliste reste associée à la gauche chilienne, à ce que fut la dernière campagne électorale de Salvador Allende, à ce que fut le gouvernement de l'Unité populaire (1970-1973). Les murs de Santiago sont ainsi investis. Sur ces murs, on écrit et on peint.

D'abord instrument de propagande, la concurrence entre brigades, et notamment entre celle des jeunesses communistes (Brigada Ramona Parra, BRP5) et celle des jeunesses socialistes (Brigada Elmo Catalán, $\mathrm{BEC}^{6}$ ), ne se donne pas sur le seul terrain du politique 
stricto sensu. Les brigades rivalisent en innovant dans le tracé, la complexité des figures, l'usage des couleurs. L'image qu'elles présentent aux passants les identifie tout autant que les messages véhiculés et que leur signature. Ainsi, chemin faisant, les brigades ouvrent-elles un espace d'expression artistique, étroitement lié à la vie politique mais suscitant, au-delà, l'attention et l'intervention ponctuelle d'artistes reconnus dont le peintre Roberto Matta.

C'est sur cette dimension artistique que s'est en partie bâtie la renommée d'une de ces brigades, la BRP. Cette renommée tient également à une manière de faire, issue de la nécessité : la vitesse. Ceci nous renvoie à une période relativement courte allant de la création de la brigade à l'élection présidentielle du candidat socialiste (1968-1970) : il fallait travailler vite pour échapper à la surveillance de la police. Dans un premier temps, ce qu'on peignait c'était des messages conçus à l'avance et destinés à un lieu précis. Au sein de la brigade, il y avait des « traceurs » chargés du contour des lettres et des « remplisseurs » chargés de colorier l'intérieur ; chaque brigadiste ayant sa couleur. Deux traceurs se plaçaient aux extrémités du mur choisi pour peindre de l'extérieur vers l'intérieur. Les premières lettres étant tracées, le remplissage commençait. Sur un mur de deux mètres de haut et de trente mètres de long la BRP écrivit un jour «Avec Allende nous vaincrons ». L'opération prit deux minutes et demie ${ }^{7}$.

Plus tard, sous le gouvernement de l'Unité populaire (1970-1973), cette expression à la fois politique et artistique fut sollicitée ouvertement par les nouvelles autorités. Des fresques entières ont alors été conçues. L'une d'elles raconta en images l'histoire du mouvement ouvrier chilien sur les rives du Mapocho à Santiago. Les méthodes de travail se modifièrent. Désormais, on pouvait prendre son temps. Puis, à partir du coup d'Etat du 11 septembre 1973, tous les partis politiques furent proscrits, les brigades interdites et leurs membres poursuivis. On entreprit d'effacer chaque peinture effectuée et de cette première étape des brigades murales, il ne resta rien. Plus tard encore, au carrefour des années 1980, et à la faveur de la réorganisation d'une opposition politique complexe ${ }^{8}$, le travail mural refit surface : sur les murs, les images renvoyaient désormais à l'histoire de la dictature, au coup d'Etat, aux crimes commis, on y voyait les visages des morts et des disparus. Depuis, les brigades se sont multipliées. Elles admettent aujourd'hui des sujets fort variés et ne sont pas nécessairement liées à des partis politiques. Une des brigades surgies à la fin des années 1980 choisit de ne plus mettre en images mais de revenir aux mots de la première période : la brigade Juan Chacón Corona, dite « la Chacón »9.

Spécificités de la brigade Chacón

6 La brigade Chacón naît en 1989 au sein du Parti communiste. Cette brigade est ainsi contemporaine du plébiscite de $1988^{10}$ et des élections présidentielles de 1989, lesquelles mettent fin officiellement à la dictature de la junte militaire ${ }^{11}$. Danilo Bahamondes, l'un des fondateurs de la BRP, sera chargé de la Chacón entre 1989 et 1997. Un différend avec le comité central l'amène alors à quitter le parti. A partir de cette année, il existera deux brigades Chacón: celle officielle du PC et celle que Bahamondes dirige en franc-tireur suivi dans cette initiative par quelques compagnons. Les repères donnés ci-dessous pour caractériser la singularité de cette brigade font fondamentalement référence à la période 1989-1997 pendant laquelle se définit: une technique d'exposition sur les murs; une calligraphie spécifique; et quelque chose comme un « parler », inédit jusque-là. 
7 «Papelógrafo ». On pourrait dire «Papierlographe »: un rouleau de papier d'environ un mètre de hauteur et de longueur variable selon l'inscription. Le plus souvent Danilo Bahamondes prend en charge le tracé et le remplissage des lettres (une seule couleur: noir sur fond blanc). Il est parfois assisté par l'un des membres de la brigade. Une fois les lettres séchées, le papier est enroulé. A l'extérieur du rouleau, on précise le contenu du message et le lieu où aura lieu le collage.

Cela se passe à la tombée de la nuit. Le nombre des présents peut varier d'un soir à l'autre (une dizaine au début des années 1990). Deux ou trois véhicules sont mobilisés. En une soirée environ dix "papelógrafos » sont disposés sur les murs de Santiago. Une fois rendus sur le lieu d'un collage, il faut - comme autrefois à la BRP - agir très vite pour leurrer les policiers. Deux ou trois brigadistes sont chargés de guetter. Au niveau du mur certains se chargent de préparer la surface (de retirer des débris éventuels, des affiches en parties décollées). Simultanément un autre passe la colle. Dès qu'un ou deux mètres sont encollés, le reste de la brigade se met en mouvement : l'un tient l'extrémité du "papelógrafo ", un autre le déroule, les autres passent leurs mains pour lisser le papier. L'ensemble de l'opération prend environ deux minutes.

Où colle-t-on? Il y a les murs réguliers. Ceux dont on sait qu'ils seront disponibles et que Danilo Bahamondes a choisis en fonction du nombre de gens susceptibles de transiter à cet endroit. Il y a également des murs mobilisés ponctuellement en fonction des chantiers de la ville et des parois disposées pour protéger lesdits chantiers. Au centre de Santiago, au moins quatre "papelográfos" sont collés sur les murs de l'Alameda (avenue principale de la capitale) ; un autre directement sur la «panaméricaine », route traversant le pays de nord à sud dont les parois sont visibles par les automobilistes et les passagers du métro. La brigade agit dans plusieurs quartiers. Un jour par semaine. Chaque quartier a son jour. D'une semaine sur l'autre les "papelógrafos » sont remplacés et toujours disposés au même endroit - exception faite des quelques espaces variables - de telle manière qu'il est possible de générer une expectative.

10 Les messages en question se distinguent ainsi par: leur support, le "papelógrafo "; leur signature (une étoile noire au bout du message, au bas de laquelle figure le nom de la brigade ; ponctuellement, en particulier en période électorale, l'étoile devient rouge et jaune) ; la calligraphie utilisée, toujours la même d'un « papelógrafo » à l'autre. Cette calligraphie est mise au service de l'objectif souhaité : que les "papelógrafos » soient lus y compris de très loin. Elle a été reprise depuis par des groupes divers liés à l'actuelle gauche chilienne - et, plus ponctuellement, aux secteurs de droite, en période électorale. Plus fondamentalement, les messages de la Chacón se distinguent par leur contenu.

11 Que dit la Chacón? Si la brigade accompagne les campagnes électorales diverses du Parti communiste sa fonction n'est pas réductible à la propagande politique. La diversité des messages est telle qu'il n'est pas possible, dans le cadre de cette présentation, de se livrer à une catégorisation satisfaisante. On peut néanmoins poser qu'il existe deux grands types de messages : les conjoncturels, étroitement liés à la vie politique chilienne et internationale ; et les autres, réflexions plus générales sur ce que devrait être la politique, la démocratie, la justice, l'économie. Le tout premier message de la Chacón a été formulé dans le cadre de l'affaire dite « des raisins ». En mars 1989, on découvre à Philadelphie qu'un chargement de fruits chiliens contient du cyanure. L'embargo est déclaré. Le ministre de l'Intérieur de l'époque, Cáceres, accuse 
ouvertement les communistes d'être à l'origine de l'empoisonnement. Dans ce contexte, la Chacón écrit sur les murs : «Cáceres miente », « Cáceres ment ». Ce message se décline sur un ton affirmatif. D'autres se présentent comme des questions. D'autres encore comme des sentences. La plupart accordent une large place à l'humour (noir, en règle générale).

Précisons avant de donner la parole que la grande majorité des phrases sont rédigées en espagnol mais il y en a eu en français, en italien et en anglais selon l'actualité commentée et les compétences linguistiques des brigadistes (ils signaient alors "Chacón polyglotte "). Il y a eu dans la brigade des hommes et des femmes. Certains d'entre eux étaient affiliés au Parti communiste. D'autres non. Il s'agissait en majorité d'adultes âgés de 30 à 45 ans. Il y en a eu aussi de plus jeunes. Durant la période 1989-1997, la brigade avait ses militants fixes auxquels se joignaient des compagnons épisodiques.

Danilo Bahamondes a la parole ${ }^{12}$

13 "Je sens qu'au sein de la Brigade Chacón nous sommes des privilégiés. Nous avons, oui, le privilège de dire ce que nous sentons, ce que nous pensons, ce dont nous sommes profondément convaincus, dans la rue (...). Quand ton objectif est de faire de la politique, ce qui est nôtre cas, car nous communiquons des idées dans la rue, il faut que tu sois bien informé. Il faut frapper juste. Il faut avoir des convictions pour ensuite être crédibles ».

14 «La brigade Chacón a été pensée pour fonctionner en démocratie, la culture militaire était alors hors-jeu... Le 'Non' avait triomphé, nous étions en démocratie. Il fallait adapter le travail (...). Nous n'avions pas de culture démocratique. Et même plus, nous ne croyions pas à la démocratie bourgeoise - c'est ainsi que nous la cataloguions. De plus, nous n'approuvions pas le type de démocratie instaurée, dans le respect de la Constitution de 1980... Ma formation était celle d'un communiste des années 1960, et les plus jeunes s'étaient formés sous la rigueur de la dictature. C'est pourquoi, nous devions apprendre à croire en la démocratie et à respecter ses codes. C'était un apprentissage ».

15 «Parmi les phrases que nous mettons dans la rue beaucoup viennent de gens importants de ce pays. Ils s'expriment dans la presse écrite et dans les journaux télévisés. Parfois, ce sont des idées compliquées ou alors exprimées de manière très longue. Alors, nous synthétisons (...). Par exemple, quelqu'un a dit : 'Les êtres humains ont besoin de faire partie d'un projet. Cela exige des idées fondamentales sur ce qu'est une société. Ce sont ces idées que nous voudrions élaborer et partager avec d'autres. Il faut réenchanter et re-signifier la politique pour qu'elle puisse tenir sa vocation d'action en vue du bien commun'. Nous, nous avons écrit sur ce sujet : 'La politique doit redevenir une pratique d'idées', 'La politique doit de nouveau interpréter des rêves collectifs'».

16 «Nous n'avons pas de concurrence, il n'y a pas d'autres brigades avec lesquelles nous puissions avoir un échange d'idées. C'est ennuyeux parce qu'il n'y a pas de pression pour nous surpasser. Si on entend une phrase intelligente d'un tel de l'UDI ${ }^{13}$, nous devons nous montrer plus intelligents. On aimerait avoir une concurrence. Mais nous constatons que les seuls à pouvoir surpasser notre travail c'est... nous-mêmes ».

Quelques messages de la Chacón

N'avez-vous pas l'impression que cette démocratie nous a été prêtée ?

[¿No le da la impresión de que esta democracia está prestada?] 
Est-ce qu'aujourd'hui le manque de courage porte le nom de raison d'Etat?

[¿Es que hoy a la falta de coraje se le llama razón de Estado?]

Empêchons que le Chili ne devienne un pays de spéculateurs

[A impedir que Chile sea un país de especuladores]

Le néolibéralisme empeste... l'air, les rêves, la vie...

[El neoliberalismo apesta... el aire, los sueños, la vida...]

Le système de santé a-t-il collapsé ou est-ce qu'il vaut que dalle, vous en pensez quoi ?

[El sistema de salud colapsó o es que vale callampa, ¿qué opina usted?] ${ }^{14}$

Maintenant les livres ne sont plus interdits par un ministre... mais par les prix

[Ahora los libros no los prohibe un ministro... sino los precios]

Un fantôme hante l'Europe. Magistrats du monde : unissez-vous ${ }^{15}$

[Un fantasma recorre Europa. Jueces del mundo uníos]

Une société grise et médiocre... mérite le foot que nous avons

[Una sociedad gris y mediocre... se merece el futbol que tenemos]

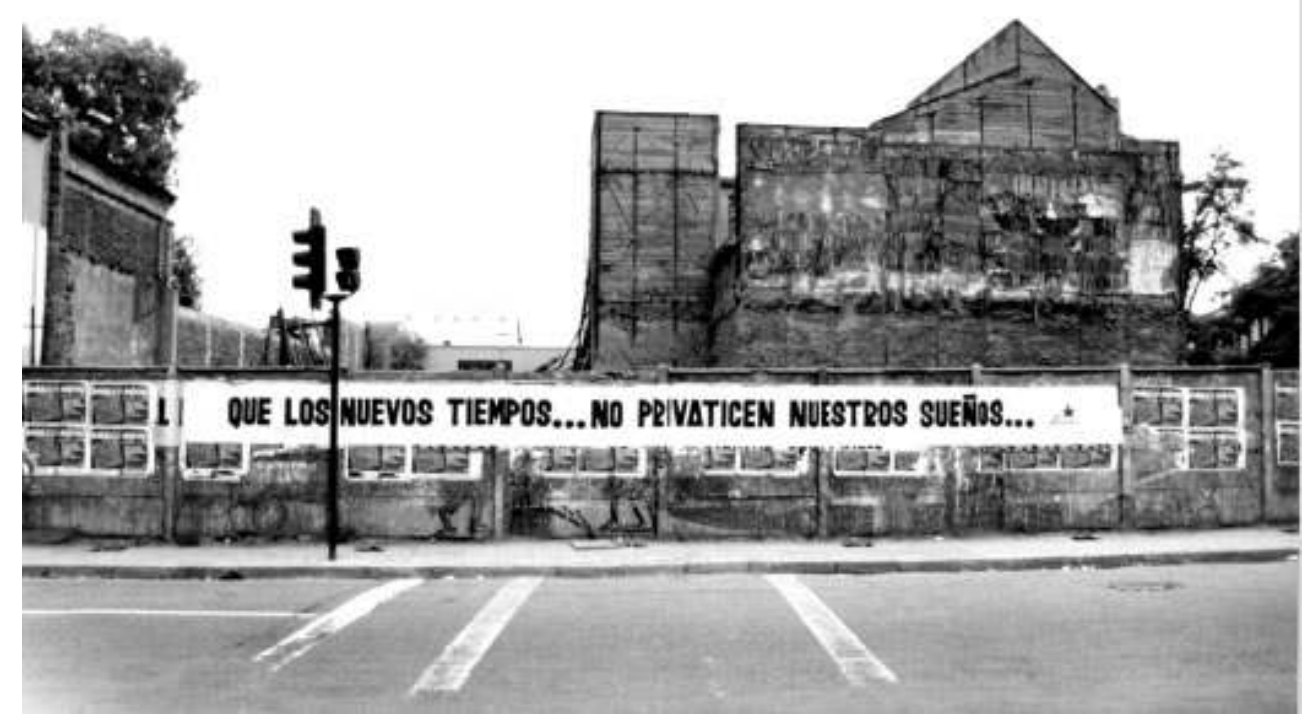

"Que les nouveaux temps... ne privatisent pas nos rêves..."

Témoignage : Alfonsina et le mur ${ }^{16}$

18 J'ai rencontré Danilo Bahamondes en 1991. J'avais 18 ans et j'étais rentrée depuis peu au Chili avec ma famille. Nous entrions alors dans la catégorie des « retornados », sorte de pendant aux « exilés ». Nous étions donc les « revenants ». Un ami espagnol était venu à Santiago faire une enquête sur les brigades murales. Je l'ai accompagné dans ses divers rendez-vous et dans l'une de ses sorties avec la brigade Chacón. Mon ami devait repartir et il n'a pas eu le temps de faire l'entretien qu'il prévoyait avec Danilo. J'y suis allée à sa place, rue Arturo Prat où la Chacón avait alors ses ateliers. J'y suis restée.

En principe, ma place n'aurait pas dû être à la Chacón mais à la BRP. A cause de l'âge. La nouvelle BRP était la brigade des jeunesses communistes. Son travail consistait fondamentalement à peindre de grandes fresques sur les murs. Ses brigadistes participaient ainsi de la vie des quartiers les plus démunis avec le soutien des habitants. Il fallait toute une matinée ou un après-midi pour peindre ces fresques. La Chacón faisait autre chose. Elle ne dessinait pas. Elle ne peignait pas l'histoire politique chilienne. Elle en commentait le présent. C'est cet ancrage dans le présent que j'ai 
choisi et j'ai choisi aussi la compagnie de Danilo, qu'on appelait «Commandant » au sein de la brigade.

On m'en avait souvent parlé comme d'une personne sortant de l'ordinaire. Avec d'autres, il avait conduit, en 1969, une marche à pied de Valparaíso à Santiago contre la guerre au Vietnam, au Laos, et au Cambodge. On disait aussi qu'il avait accroché un drapeau vietnamien dans un bateau nord-américain et qu'on l'avait enfermé pour cela dans une grotte. Surtout, il était une figure emblématique de la première BRP. L'homme au casque jaune. Ce casque jaune, caractéristique des ouvriers du bâtiment, était le signe distinctif des brigadistes de la BRP. Ce n'était pas une coquetterie. Le casque permettait de protéger la tête lors des affrontements avec les carabiniers. On peut en contester l'efficacité et pour preuve, ce souvenir de ma mère, militante des jeunesses communistes à l'époque :

21 Les dirigeants du PC et ceux de la BRP avaient proposé que les murs soient pris d'assaut le 4 septembre 1970: chaque jeune communiste, accompagné d'un brigadiste " professionnel ", s'en est allé peindre les murs. Santiago fut pour ainsi dire totalement revêtue de couleurs en ce jour où Salvador Allende remporta les élections présidentielles. C'est coiffé d'un casque, et sous le casque, les bandages... que Danilo s'est posté devant son mur.

En 1991, il devait avoir une quarantaine d'années. Si j'examine mes propres souvenirs, je dirais que c'était un homme inventif, souvent irrévérencieux, irréductible. C'est aussi ce que transmettaient les messages de la Chacón en ce début des années 1990 et ce n'était pas rien.

Je ne peux pas me prononcer pour les autres mais je me souviens de ce retour au Chili et des années 1990 en général comme d'une période de grande tristesse. Je sentais que tous mentaient. Les hommes politiques, les journalistes, les professeurs au lycée puis à l'université, et même, l'homme de la rue. Le président de la République était alors Patricio Aylwin, démocrate-chrétien. Il était celui qui avait soutenu le coup d'Etat de 1973 et son repentir tardif ne changeait rien à l'affaire ${ }^{17}$. C'était l'époque où les anciennes autorités de la dictature pouvaient s'exprimer en toute impunité et dire que telle chose n'avait pas eu lieu. Ce fût le cas de Contreras notamment. L'ancien directeur de la police secrète, la DINA. Dans une de ses déclarations, il avait nié les prisons secrètes, la torture et les disparus. A l'entendre, on aurait rêvé. L'histoire chilienne de 1973 à nos jours était un cauchemar, rien de plus. Il y avait aussi les manifestations en soutien à la libération des prisonniers politiques de la dictature dont certains n'avaient pas encore été libérés. Cela se finissait souvent en altercations avec les carabiniers et j'ai entendu un jour un passant hurler: "Qu'est-ce que vous voulez! Le retour des militaires?». Je ne savais pas s'il avait fait, lui, quelque chose pour chasser les militaires. Mais c'était le cas des prisonniers politiques au nom desquels nous étions sur les lieux.

24 Dans ce contexte, le travail de la Chacón prenait du sens. Sans négliger le caractère novateur de la technique, son aspect esthétique aussi - car ces lettres immenses habitaient les murs de la ville et sont devenues caractéristiques d'un certain paysage urbain : l'aspect central était donné par la composante politique. Du fait même de coller directement sur les murs, ce qu'on contestait c'était le monopole de la prise de parole. Dans une société où les médias appartiennent aux classes dominantes, où ils soutiennent la politique de l'autruche, et où l'hypocrisie fait rage, écrire sur un mur des phrases qu'aucun journal n'accepterait de publier (sauf ceux d'extrême gauche que presque 
personne ne lit) c'était un geste hautement politique. Un geste entrepris par les bases. L'écriture sur les murs ce n'était pas l'affaire des élites, pas mêmes des élites communistes. Les brigadistes ne sortaient pas de la Sorbonne. A ce propos, il y a une anecdote. J'entamais à l'époque des études universitaires. Au sein de mon cursus, on devait suivre des cours d'économie. Je les séchais avec assiduité pour m'en aller à l'atelier peindre les papelógrafos avec Danilo. Un jour, il m'a demandé ce que je faisais comme études:

- Histoire.

- Histoire ? C'est bien. Vous serez une chômeuse cultivée.

Il y avait des chômeurs au sein de la Chacón. Des mères de famille aussi. D'autres avaient des activités variées. On se connaissait plus ou moins, selon les affinités. On était plus mystérieux au sujet des noms. Chacun avait été rebaptisé d'office. C'était une vieille habitude liée à la clandestinité. Un soir, nous nous sommes fait arrêter et il a fallu sortir les cartes d'identité. Ce qu'on a pu rire en découvrant nos vrais prénoms... Dans mon cas, le baptême est venu d'une erreur. Au cours d'une sortie, le Commandant m'a appelée « Alfonsa » :

- Je ne m'appelle pas Alfonsa... Qu'est-ce que c'est que ce prénom?

- Mais c'est un très joli prénom. Il y a même une chanson...

- La chanson s'appelle " Alfonsina et la mer », Commandant...

- Ah, oui. Alfonsina. Va pour Alfonsina, comme la poétesse ${ }^{18}$.

Les sorties n'étaient pas tristes. On était content d'y être. Il y avait un camarade, on va dire V., qui avait un certain penchant pour la boisson. Ça lui prenait le soir, uniquement le soir. Mais à l'heure de la sortie, il était déjà gris. Alors souvent le Commandant l'empêchait de venir. C'était toute une histoire. Notre brigade, celle du centre de Santiago, collait le mardi. Chaque mardi, la même chose. V. qui s'approche des voitures, qui crie qu'il veut sortir lui aussi, qui s'accroche à la camionnette et les camarades lui décollent les doigts, et il finit par lâcher prise et reste au milieu de la chaussée. Mais de temps en temps, il venait. C'était un ami de Danilo, de l'époque de la BRP. Je me souviens qu'une fois, il s'est mis à pleurer dans la voiture, il a mis sa tête sur mon épaule et il a pleuré. Il disait : « ah !... Alfonsina, si tu savais, on venait du monde entier pour nous voir... même Guayasamin est venu au Chili ». La BRP de sa jeunesse c'était toute sa fierté.

La Chacón aussi était motif de fierté. Pour les brigadistes et à certains égards pour les dirigeants du Parti communiste. C'est qu'il n'y avait pas d'équivalent au Chili. Il n'y en a jamais eu par la suite. Les papelógrafos de la Chacón étaient conçus comme un média alternatif. Ce qu'on voulait c'était faire entendre un autre discours et que ce discours, cette pensée, cette réflexion parviennent au plus grand nombre possible. La plupart des messages étaient l'oeuvre de Danilo. Il était pourtant preneur de toutes les bonnes idées. Seulement, de bonnes idées, nous n'en avions pas tous les jours alors qu'il était prolifique. Indiscutablement, le fait que Danilo ait été le chef de la brigade, sa principale autorité (même si le mot autorité aurait fait rire le bonhomme) donnait le ton. Il avait une manière bien à lui de penser les écrits, de les élaborer et maniait l'ironie comme personne. Les expressions utilisées étaient quelque peu codifiées et avaient souvent recours à l'argot. Il y avait un « style Chacón ».

Parmi les messages, ma préférence allait à ceux qui revendiquaient le mot politique. Là encore, c'était important à cause de la déroute. Les hommes politiques professionnels étaient les otages des militaires. Beaucoup d'entre eux étaient pris du syndrome de Stockholm. L'extrême gauche n'était plus une force politique significative. Chez les 
jeunes, cette déroute était aussi visible. Bien sûr, tous les partis avaient leurs " jeunesses ", à gauche, à droite, et au centre. Mais, d'autres jeunes ne savaient pas toujours où s'immiscer. Sans trop généraliser, j'ai tendance à penser que ma génération (et j'entends par là, les «enfants » de l'Unité populaire) a été caractérisée par le très grand respect qu'on éprouvait pour les «vieux». Car ces vieux, nos parents, constituaient un exemple. Un exemple que l'on ne savait pas toujours comment suivre dans le contexte politique auquel nous avions affaire. Cela peut sembler naïf mais il s'est trouvé des jeunes pour choisir la lutte armée dans les années 1990. Certains, parmi les plus âgés, et qu'on appelle "la génération des années 1980 ", avaient assumé des fonctions politiques. Mais, les jeunes des années 1990 (que, de fait, on n'a pas appelé la "génération des années 1990 ", c'est une génération sans nom particulier) avançaient dans le noir et il n'était pas du tout clair que cette chose politique, que l'on pouvait observer à travers les médias, ait un quelconque lien avec ce qu'avait été la politique du temps de nos parents. Alors, quand la Chacón écrivit sur les murs « Que les nouveaux temps... ne privatisent pas nos rêves... », je me suis sentie pleinement concernée.

Pourtant, à côté de ce sentiment de fierté, la Chacón était aussi l'objet de critiques. Parfois, elles étaient d'ordre disciplinaire. Les dirigeants du Parti communiste n'étaient pas informés au préalable des messages qu'on allait coller. Or, la Chacón était clairement identifiée par le public comme étant du PC. Ce qu'elle était effectivement mais de manière bien moins organique qu'on n'aurait pu le penser. De telle sorte, qu'il $\mathrm{y}$ avait parfois des divergences sur l'opportunité de tel message.

30 Je voudrais ouvrir une parenthèse pour clarifier les liens de la brigade avec le PC. Il s'est dit beaucoup de choses depuis la rupture de 1997. Cette année-là Danilo s'éloigna du parti et il y eut une scission au sein de la Chacón. Outre les dénigrements dont Danilo a personnellement fait l'objet au sein du parti (avec de respectables exceptions), certains ont cru bon de souligner que la brigade Chacón ne faisait que retranscrire les positions et les consignes du PC. Ceux-là mentent. Mon expérience parle d'autre chose. Les liens entre le PC et la Chacón (même si le PC finançait et même si ses dirigeants pouvaient donner des orientations) étaient étanches. D'où très précisément, les rappels à l'ordre. Il fut une époque où le PC chilien était une force politique capable de promouvoir le changement, de susciter l'enthousiasme de la jeunesse et d'éveiller en elle ce qu'elle avait de plus créatif. La BRP témoigne aussi de cela. Ce n'était plus le cas au tournant des années 1990. Le PC luttait alors pour sa survie et peinait très précisément à trouver ses mots, à se faire entendre, à élaborer une pensée crédible du changement politique. Il m'en coûte de le dire, car je crois encore aujourd'hui que le PC chilien, même diminué, même cherchant le chemin et ne le trouvant pas, a joué un rôle important durant ces années-là. Ce fut l'acteur borné, refusant d'accepter tous les pactes indignes que les élites de la Concertation nous proposaient. Que Danilo Bahamondes ait fait une analyse différente à un moment de sa vie : ça le regarde. Cela ne saurait compromettre à posteriori ce qu'a été son rôle au sein de la brigade du temps où elle était communiste.

31 Et du temps où elle était communiste, ce qu'on nous contestait également c'était quelque chose comme un manque de tenue. Outre l'anecdote de V., les bouteilles de pisco étaient parfois de la partie. C'était le propre de quelques camarades, pas de tous, de quelques uns. Personnellement, je n'ai jamais rien trouvé à redire. La seule question importante pour moi c'était « Le travail est-il fait? Oui ? Non? » Oui, il l'était. Certains camarades de la Chacón aimaient le pisco, le foot et les séries télévisées, surtout une 
série brésilienne qui passait à l'époque, il y avait une actrice très belle dans ce téléfilm. Autrement dit, ces camarades de la Chacón étaient comme $90 \%$ des Chiliens. Ils faisaient en plus de la politique, à leur manière, certes. Mais aux yeux d'autres militants, peut-être plus "orthodoxes", ils n'étaient pas des militants comme les autres. Selon les occasions, on les présentait comme des pionniers, des braves, ou comme des militants de second ordre. Puis, il y avait cette image de la brigade "groupe de choc ». En ce sens que les militants n'étaient pas des intellectuels et qu'ils étaient parfois prompts à la bagarre. La brigade était un groupe de choc. Dans un sens noble. Elle innovait tous les jours et elle assumait parfois d'autres tâches que les siennes dont beaucoup se félicitaient. C'est un militant de la Chacón, dit «le loup-garou », qui le 11 septembre 1992 a éteint la «flamme de la liberté » instaurée par les militaires pour célébrer le coup d'Etat de 1973.

Autre aspect qu'il est important de retenir c'est que le travail de la Chacón était un travail relationnel. Et ce à différents niveaux. Danilo rassemblait. Avoir la volonté d'être ensemble, après tant d'années de dispersion, c'était en soi un acte de force. Les jours de collage, on arrivait toujours en avance pour préparer le café et discuter de politique, mais aussi de tout autre chose, des soucis quotidiens, d'un livre qu'on avait lu, ébaucher un pas ou deux de salsa. Puis, à un autre niveau, il y avait la rue. C'est peut-être parce que j'avais longtemps vécu hors du Chili, le fait est que c'est auprès de la Chacón que j'ai compris qu'un pays, c'est fait de beaucoup de rues et de tout autant de murs. Ces murs m'appartenaient parce que j'en connaissais la surface, j'y avais posé mes mains, j'en avais retiré les clous. « Ce pays est aussi le nôtre. Nous avons notre mot à dire ». C'était ça, l'élan de la Chacón. Puis, dans les rues, il y avait les passants.

Peu à peu les gens prenaient l'habitude. Coller sur les murs ces immenses rouleaux de papier, c'était devenu normal. Mais une fois, je n'ai assisté à ça qu'une seule fois, les gens qui étaient là et qui attendaient le bus ont applaudi... Je n'ai plus aucune idée de ce qu'on avait collé à cet endroit, mais ils ont applaudi et ça nous a fait drôle. On ne cherchait pas à discuter avec les passants. On n'avait pas le temps. Il y avait toujours la possibilité de se faire arrêter, on agissait donc très vite. Mais ce petit échange a eu lieu au moins ce soir-là. Les autres échanges c'était avec la police et ils étaient moins sympathiques. Cela se finissait souvent en course. Sauf une fois. Un carabinier nous a interpellés. C'était l'un de ces carabiniers que nous appelions les "tortues ninjas" parce qu'ils étaient en moto et qu'ils portaient sous leur casque une sorte de cagoule verte. Le flic s'approche, nous demande ce que nous faisons là, nous expliquons. Le type lit ce qu'il y a sur le mur et là... Il enlève le casque, la cagoule et nous dit : « vous voyez, je vous montre mon visage, je suis d'accord avec ce que vous avez écrit ». On a été pris au dépourvu. C'était du jamais vu.

Plus généralement, les papelógrafos de la Chacón ne laissaient pas indifférents. D’une part, ils étaient bien faits. Il faut tout de même le dire. Il suffit de comparer avec les nouveaux. « Non à l'APEC », «Bush : dehors ». On pourrait dire : « façon Chacón ». Sauf que le tout est écrit en lettres rouges, trop petites et bien serrées. La calligraphie ne suffit pas. Il n'y a pas de cause à effet. Les papelógrafos de la Chacón « accrochaient ». J'ai souvent entendu, dans les lieux les plus divers, «tu as vu le dernier papelógrafo de la Chacón? », comme on aurait dit : «tu as lu l'article d'un tel ?». J'ai aussi entendu de dures critiques à l'égard de certains messages et notamment à propos de la remise en question de la classe politique en tant que telle. Peu importe. L'objectif était de 
communiquer, d'interpeller, de faire parler, de générer des discussions. Cet objectif a été atteint.

Quand je regarde aujourd'hui les nouveaux écrits sur les murs, mon sentiment est partagé. Peindre sur un mur, c'est une petite victoire. Il y a ce côté obstiné. Que les partis de droite se soient saisis de la technique ne change rien. Ça leur passera. Ça leur passe déjà. Reste la volonté de laisser une trace; de donner son avis aussi, quand personne ne vous le demande. Mais je ne peux m'empêcher de traquer les murs. Je ne peux m'empêcher de chercher les mots de la Chacón que j'ai connue. C'est qu'il ne reste rien ou si peu. Danilo avait pris la précaution de photographier les papelógrafos, semaine après semaine. Il s'agit d'une collection personnelle. Les gens n'y ont pas accès. On ne sait pas vraiment qu'elle existe. Le PC n'en a rien fait. Du moins à ma connaissance. D'autre part, le passant ordinaire n'associe pas le nom de Danilo Bahamondes à cette ancienne brigade. Cela est d'autant plus vrai pour les autres brigadistes. Nous n'avions pas de nom. Notre nom à nous c'était « Chacón ».

$\mathrm{Au}$ mois d'août 2001, quelqu'un m'a appelé pour m'annoncer de vive voix que Danilo était mort, qu'il s'était étalé sur un trottoir. Depuis, Santiago n'est plus la même. Il y a cette perte au sein de la vie politique. Puis, les relations personnelles. Le mur que l'on porte en soi. Celui qu'on longe aussi en pensant à la petite phrase insidieuse qu'on aurait bien envie d'écrire. Il m'arrive de relire un mot que Danilo m'avait envoyé alors que je me trouvais de nouveau à l'étranger, histoire de parfaire mon avenir de chômeuse cultivée. Ce mot disait : «Du Chili je ne te dirai rien pour ne pas t'attrister. Mais j'éprouve un grand amour pour ce que je fais et les peines et les désillusions glissent sur moi ». Comme sur le mur.

Annexe : Propos du peintre Roberto Matta

Tu as travaillé au Chili avec la brigade Ramona Parra...

A vrai dire, c'était une manifestation de solidarité, d'amitié. Je me souviens d'une fois, on était à La Granja, j'avais organisé les couleurs : un tel était le vert, un autre le rouge, un autre le jaune, un autre le blanc, un autre le noir. Chacun avec son pot de peinture. Ça se passait dans une piscine. J'avais besoin du rouge et le rouge était dans la piscine. Je l'engueulais parce qu'il me fallait absolument le rouge et il fallait attendre parce qu'il était en train de se sécher. Et le vert, par exemple, il était en train de faire de la balançoire ou en train de boire une bière. Le bleu était bien là mais ce n'était pas du bleu que je voulais. Alors il fallait que j'utilise le bleu au lieu du vert. C'est comme ça que, pour finir, les drapeaux rouges étaient bleus. Peut-être que, ce n'est pas impossible, je leur ai donné un peu d'humour, et c'est avec cet humour, partie intégrante de la poésie, qu'on peut se réveiller pour voir certaines choses (...).

Il me paraît important de contester la réputation que certains essaient de donner à ces jeunes du peuple. Les brigades avec lesquelles j'ai travaillé, la brigade Ramona Parra, par exemple, sont un modèle de travail volontaire. Ces jeunes n'ont pas plus de dix-sept ans ; ils peignent en dehors des heures d'étude ou de travail. Ils sont en train d'essayer de mettre en place une sorte d'université populaire. A propos des problèmes, par exemple, de l'art. J'ai discuté avec eux. Ces brigades essayent d'apprendre le plus qu'elles peuvent et elles exercent. Le grand problème des universités bourgeoises c'est qu'elles ne donnent pas un langage pour se comprendre avec les travailleurs. Il y a au sein des brigades une volonté d'université populaire (...). 
Sources - Conversation avec L. Guastavino et G. Torres, revue Araucaria, 1978; Conversation du peintre Roberto Matta avec les élèves des Beaux Arts. Instituto de Arte Latinoamericano. 25 octobre 1971. Enregistré par E. Saúl. Voir : http://www.abacq.net/ imagineria/arte2.htm

\section{NOTES}

1.. Sarmiento F. Domingo, Recuerdos de Provincia, Buenos Aires, Emecée Editores, 1998 (1ère édition 1850), p. 17. Edition préfacée et annotée par Jorge Luis Borges. L'écrivain fait ici référence à Appelle de Clos, portraitiste d'Alexandre le Grand.

2.. Le lecteur trouvera une sélection d'images sur le site Internet. Voir : http:// conflits.org

3.. Voir sur le sujet, le numéro spécial « Painting politics » de Social Alternatives, Vol. 20 , No. 4 , octobre 2001, dirigé par Roland Bleiker.

4.. Ce dossier prend appui sur un ouvrage principal : Sandoval A., Palabras escritas en un muro. El caso de la brigada Chacón, Santiago, Ediciones Sur, 2001. Il a été effectué par une jeune sociologue chilienne et comprend une bibliographie importante. On peut s'y rapporter pour en savoir plus sur les antécédents non chiliens de la peinture sur les murs et ses liens avec le politique. Voir également : Kaulen V., Martínez B., Sepúlveda J., "Análisis comprensivo de la construcción de un discurso crítico de la modernidad chilena : brigada Chacón », Universidad Diego Portales, Escuela de Periodismo, Santiago, 2003.

5.. Ramona Parra : jeune militante communiste morte aux mains de la police suite à une arrestation en 1946.

6.. Elmo Catalán : journaliste chilien, membre du ELN (Ejército de Liberación Nacional ; Armée de Libération Nationale) section chilienne et du ELN bolivien. Il est assassiné en Bolivie en 1970.

7.. Voir témoignage de Luis A. Corvalán in Sandoval A., op. cit., p. 30.

8. Dans le milieu des années 1980, un dialogue s'instaure progressivement entre autorités militaires et forces d'opposition d'abord réunies au sein d'une « Alliance démocratique » puis au sein de la "Concertation des partis politiques pour le 'Non' » (en référence au plébiscite de 1988) puis " pour la démocratie » (à l'occasion des élections présidentielles de 1989). Les principaux acteurs de cette opposition sont le Parti Démocrate-chrétien et le Parti socialiste - farouches adversaires sous le gouvernement de l'Unité populaire.

9.. Juan Chacón Corona (1896-1965) : ouvrier d'origine paysanne, militant du Parti communiste depuis sa fondation en 1922.

10.. Lors du plébiscite du 5 octobre 1988, le « Non » (à la poursuite du gouvernement militaire) l'emporte avec $56 \%$ des voix versus $44 \%$ des voix favorables au « Oui ».

11.. Les élections présidentielles ont lieu en décembre 1989. Le président sortant, leader de la "Concertation ", est investi en mars 1990. En dépit de ces élections libres les autorités civiles doivent composer au jour le jour avec les autorités militaires en vertu de la Constitution de 1980 et d'une série de mesures adoptées à la fin du 
gouvernement militaire. Entre autres, la Constitution de 1980 proclame que les forces armées sont les garantes des institutions chiliennes. Bien qu'amendée sur certains points, cette constitution détermine les limites de la démocratie chilienne et le rapport des forces entre autorités civiles élues et autorités militaires de fait - plus ou moins présentes dans la vie politique chilienne selon l'agenda politique des différents gouvernements qui ce sont succédés depuis 1989.

12.. Extraits d'entretiens cités dans l'ouvrage de Sandoval A., op. cit.

13.. Union démocratique indépendante. Parti politique d'extrême droite.

14.. «Vale callampa », expression très populaire, cela ne vaut rien (« callampa » : champignon).

15.. Référence à l'arrestation du général Augusto Pinochet à Londres en octobre 1998.

16.. Témoignage d'une brigadiste, traduit de l'espagnol (Santiago, novembre 2004). Alfonsina a travaillé à la Chacón pendant un peu plus d'un an.

17.. En 1990, une commission gouvernementale est chargée d'enquêter sur les crimes commis sous la dictature. Les résultats de cette enquête sont présentés publiquement par Patricio Aylwin à la télévision (mars 1991). A cette occasion, le président de la République demande pardon aux victimes en tant que plus haut mandataire de la nation.

18.. Alfonsina Storni, poétesse argentine (1982-1938). La chanson « Alfonsina y el mar », d'Ariel Ramirez et Felix Luna, lui est dédiée.

INDEX

Mots-clés : art, murs, regards sur l'entre-deux, politique

Index géographique : Chili

Thèmes : brigade Chacon

\section{AUTEUR}

\section{ANTONIA GARCIA CASTRO}

Antonia Garcia-Castro est docteur en sociologie, co-rédactrice en chef de Cultures \& Conflits 